\title{
Regards sur la formation des maîtres en France
}

A view of teacher training in France

Observaciones sobre la formación de los profesores en Francia

\section{Emmanuel Fraisse}

\section{CpenEdition}

\section{Journals}

Édition électronique

URL : http://journals.openedition.org/ries/940

DOI : $10.4000 /$ ries.940

ISSN : 2261-4265

Éditeur

Centre international d'études pédagogiques

Édition imprimée

Date de publication : 1 décembre 2010

Pagination : 61-72

ISBN : 978-2-8542-583-1

ISSN : $1254-4590$

Référence électronique

Emmanuel Fraisse, "Regards sur la formation des maitres en France», Revue internationale

d'éducation de Sèvres [En ligne], 55 | décembre 2010, mis en ligne le 01 décembre 2013, consulté le 20 avril 2019. URL : http://journals.openedition.org/ries/940 ; DOI : 10.4000/ries.940 


\section{Regards sur la formation des maîtres en France}

\section{Emmanuel Fraisse}

En France comme dans tous les pays comparables, la question de la formation des maîtres et de son évolution constitue un défi majeur : il en va en effet de la qualité et des capacités d'adaptation du système éducatif, dans un monde où les connaissances et leur transmission sont devenus des enjeux décisifs, tant au plan économique que social et moral. À l'heure où le système de formation des maîtres connaît des transformations importantes, le poids de l'histoire éducative de la France, le rôle social et symbolique de l'école dans ce pays, l'impact de la tradition des concours de recrutement des enseignants sur la vie universitaire et le rôle décisif et direct que joue l'État dans ce domaine rendent la situation particulièrement complexe dans un contexte d'autonomie accrue des universités.

\section{Primaire et SECONDAIRE}

Ainsi que l'a montré Antoine Prost il y a maintenant plus de quarante ans ${ }^{1}$, il est difficile de comprendre la situation éducative française d'aujourd'hui sans avoir à l'esprit la disposition fondamentale qui a structuré ce système à travers l'œuvre de la Troisième République. À une école de masse vouée à inculquer aux couches populaires des savoirs de base (lire, écrire, compter, comprendre du monde et de son histoire ce qui est nécessaire pour se comporter en citoyen) s'est longtemps opposée une formation d'élite destinée aux enfants des couches supérieures et aux plus méritants, aux plus chanceux et aux plus susceptibles d'adaptation des enfants du peuple. D'un côté, l'école communale et le monde des instituteurs, de l'autre le lycée et le monde des professeurs; à l'univers du primaire s'opposait celui du secondaire : les laboureurs d'un côté, les orateurs de l'autre. Qu'on relise Camus et Le premier homme ${ }^{2}$ pour se faire une idée de ce que pouvait représenter, dans les années trente, le passage d'un univers à l'autre pour un petit Blanc pauvre d'Alger, orphelin de guerre de surcroît et fils d'une Espagnole illettrée. Ou, quelques années plus tard et dans un contexte différent, mais qui montre bien la complexité du fonctionnement de «l'ascenseur social» dans le monde des instituteurs, qu'on se reporte aux souvenirs et réflexions de Mona Ozouf dans Composition française ${ }^{3}$.

\footnotetext{
1. Antoine Prost, Histoire de l'enseignement en France, 1800-1967, Paris : Armand Colin, 1968; nouv. éd. 1983.

2. Albert Camus, Le premier homme, Paris : Gallimard, "Cahiers Albert Camus 7 », 1994 ; nouv. éd. « Folio », 2000.

3. Mona Ozouf, Composition française, retour sur une enfance bretonne, Paris : Gallimard, 2009.
} 
Si l'on observe les choses de plus près, il y avait bien en France, jusqu'à la Seconde Guerre mondiale, deux systèmes pratiquement étanches, chacun disposant d'une filière autonome et complète : il existait un "primaire " du secondaire (les "petites classes des lycées »), un « secondaire » du primaire (les cours primaires supérieurs), un "supérieur " du primaire consacré à la formation des professeurs des écoles normales d'instituteurs alors que le supérieur du secondaire (les classes préparatoires aux grandes écoles, l'université et les grandes écoles) commençait après le baccalauréat.

Très naturellement, compte tenu de l'importance des questions scolaires dans la vie politique, sociale et morale de la France, cette bipartition se trouvait reproduite dans le système de la formation des maîtres, qui a d'emblée été ressenti comme un enjeu central et, pour tout dire, une affaire de l'État, dans laquelle le dispositif du concours de recrutement joue un rôle décisif et fait de chaque enseignant du secteur public un fonctionnaire d'État et aux yeux de beaucoup, hier comme aujourd'hui, assure le caractère "national » du système éducatif français et sa justification méritocratique.

Outre un système spécifique de diplômes (le certificat d'études primaires pour l'une, le baccalauréat pour l'autre), chacune des deux filières avait donc son propre mode de formation des maîtres, tous deux fondés sur des concours de recrutement, les écoles normales d'instituteurs jouant un rôle décisif pour l'École, l'Université s'étant vu attribuer la formation des professeurs des lycées. L'existence de deux types d'écoles normales supérieures parachevait cette construction : à celles de Saint-Cloud (garçons) et de Fontenay-aux-Roses (filles) revenait la formation des futurs professeurs des écoles normales d'instituteurs ; à celles de la rue d'Ulm (garçons) et de Sèvres (filles), il incombait de recruter et de former l'élite des professeurs de lycée et des universités. La différence entre les deux systèmes n'était pas seulement qualitative, elle était aussi criante au niveau des effectifs : à la veille de la Deuxième Guerre mondiale, on comptait 132000 instituteurs pour 15000 professeurs de lycée ${ }^{4}$.

Sous la pression de la montée en puissance de la formation de masse ou, comme on voudra, de la démocratisation scolaire, l'action éducative de la $V^{e}$ République s'est confondue avec une unification progressive des deux systèmes naguère distincts et quasi étanches. Désormais, l'école élémentaire n'est plus que le premier échelon d'une organisation qui relève beaucoup plus du cylindre que de la pyramide : tous les élèves ont vocation à parcourir la totalité du parcours devenu commun, au moins jusqu'à la fin de la scolarité obligatoire ${ }^{5}$. Tel est d'ailleurs, au moins sur le plan des principes, le sens de la proclamation datant

4. Voir Jérôme Gautheret et Thomas Wieder, "L'instituteur, voilà l'ennemi ", in : Histoire(s) de l'été 40, Le Monde, $1^{\mathrm{er}}$ août 2010, p. 14. On gardera à l'esprit qu'une des caractéristiques actuelles de l'enseignement primaire est de scolariser les enfants à partir de 3 ans, voire de 2 ans : au-delà de la démographie et de la réduction des effectifs d'élèves par classe, l'extension de l'école maternelle explique pour une large part l'augmentation du nombre d'instituteurs en France.

5. En effet, après le collège, près de $44 \%$ des élèves sont orientés vers des filières professionnelles. 
de près de vingt-cinq ans déjà de l'objectif de " $80 \%$ d'une classe d'âge au niveau du bac ", alors que c'était $6 \%$ en 1940 et qu'à la même époque, il y avait moins d'étudiants qu'on ne relève aujourd'hui d'inscrits en doctorat (70 000). Et, compte tenu des classes d'âge concernées, on compte aujourd'hui plus de professeurs dans le second degré que dans l'enseignement élémentaire, respectivement 520000 et 360000 , secteurs public et privé confondus ${ }^{6}$.

\section{LES IUFM : \\ UNIFIER ET UNIVERSITARISER LA FORMATION DES MAÎTRES}

La création, au début des années quatre-vingt-dix, des instituts universitaires de formation des maîtres (IUFM), à la suite de l'adoption de la Loi d'orientation sur l'éducation du 10 juillet 1989 (dite loi Jospin) répondait en effet pour partie à l'ambition parallèle d'unifier et d' " universitariser » l'ensemble de la formation des maîtres.

Unifier, c'est-à-dire avant tout rassembler dans un même établissement, sous l'impulsion et le contrôle de l'État, les formations des maîtres de l'enseignement primaire, de l'enseignement secondaire et de l'enseignement technique et professionnel de chaque académie ${ }^{7}$. Ainsi les anciennes écoles normales d'instituteurs (ENI), les centres pédagogiques régionaux (CPR) et les écoles normales nationales d'apprentissage (ENNA) voient leurs personnels, et très souvent leurs locaux, rassemblés dans les nouveaux IUFM. Unifier encore, et en un sens universitariser, en faisant de la licence le diplôme nécessaire pour se présenter à l'ensemble des concours de recrutements du corps enseignant. Sur un plan matériel et symbolique, la barrière entre instituteurs et professeurs est abolie : les carrières et rémunérations sont identiques pour la plupart et tous les candidats recrutés et formés dans les IUFM relèvent de la catégorie " A » de la fonction publique et sont désormais tous "professeurs" : professeurs des écoles, professeurs de lycées et collèges, professeurs de lycées professionnels. On le voit : la formation des maîtres a constitué un des leviers essentiels de la réalisation des ambitions de la Loi d'orientation de 1989, dont l'article premier proclame que "l'éducation est la première priorité nationale ».

Second objectif de la création des IUFM : universitariser. Cette universitarisation est restée partielle jusqu'à une date récente, malgré l'affirmation initiale de la vocation de ces établissements à participer à la recherche en

6. Chiffres 2006. Source : ministère de l'éducation nationale, Note d'information février 2007, http://media. education.gouv.fr/file/42/9/4429.pdf. Cette modification quantitative en faveur du poids de l'enseignement secondaire a eu un effet visible au plan syndical. Lorsque les professeurs du second degré sont devenus plus nombreux que les instituteurs, les équilibres idéologiques et politiques au sein de la Fédération de l'éducation nationale (FEN) ont été transformés, expliquant pour une large part la scission de cet organisme.

7. On compte aujourd'hui trente académies, et moins en 1989 puisque Antilles-Guyane constituait alors une académie unique. 
éducation. En effet, les IUFM, institués par la loi de 1989 comme établissements publics administratifs, ne bénéficiaient pas du statut des universités et des autres établissements publics à caractère scientifique culturel et professionnel. Notamment, ils n'étaient pas habilités à décerner des diplômes et, plus accessoirement, leurs directeurs n'étaient nommés qu'après approbation du ministre. C'est que l'État, employeur et recruteur, n'entendait pas renoncer à son rôle spécifique dans le domaine de la formation des maîtres. Ainsi ce sont les recteurs d'académie qui, jusqu'en 2007, présidaient les conseils d'administration des IUFM et c'est le ministère de l'Éducation nationale qui, en tant qu'employeur, continue à valider la qualification professionnelle des étudiants stagiaires à la fin de leur parcours de formation professionnelle d'une année. Universitarisation limitée, enfin, puisque les enseignants-chercheurs de statut universitaire sont longtemps restés très minoritaires parmi les personnels de ces nouveaux établissements qui, pour une large part, et notamment en ce qui concerne la formation des professeurs des écoles et les professeurs d'établissements techniques et professionnels, étaient pour l'essentiel constitués de professeurs relevant du second degré déjà en place. De surcroît, les carrières des enseignants chercheurs recrutés dans les IUFM ont pu, dans bien des cas, être affectées par une méfiance certaine manifestée par le reste de la communauté universitaire à leur égard.

Dans les faits, la division de la formation professionnelle par ordres d'enseignement à laquelle les IUFM entendaient remédier s'est perpétuée. La formation professionnelle d'une année succédant à la réussite aux épreuves théoriques des différents concours est pour l'essentiel restée centrée sur le degré d'enseignement concerné et n'a offert que très peu d'éléments de tronc commun. De plus, alors que dans la plupart des cas les futurs professeurs du second degré préparaient le concours du certificat d'aptitude au professorat de l'enseignement secondaire (CAPES) dans les universités, une très grande partie de ceux du premier degré étaient admis à préparer pendant une année le concours de professeurs des écoles dans les IUFM eux-mêmes. Ainsi le même IUFM pouvait-il être exclusivement voué à la formation professionnelle pour le second degré et dans le même temps fonctionner comme lieu de préparation au concours et de formation professionnelle pour le premier degré.

Reste l'essentiel : avec les IUFM, le métier d'enseignant est bien considéré comme dépendant d'une formation professionnelle qui, après les épreuves théoriques du concours de recrutement, fait alterner cours théoriques et stages dans des classes et débouche sur un mémoire professionnel en liaison avec l'ensemble de ces expériences. Si cette dimension professionnelle était déjà présente depuis fort longtemps dans la formation des instituteurs, il s'agit d'une modification majeure en ce qui concerne les professeurs de lycées et collèges. En effet, la dimension professionnalisante des anciens CPR était très inégale, souvent superficielle et très largement fondée sur une initiation au métier et à ses ficelles par un système qui relevait avant tout d'un compagnonnage débonnaire. 
Car en définitive, c'était bien le concours et ses épreuves théoriques qui étaient décisifs et non l'année de stage de CPR : le primat revenait à la constatation des connaissances acquises par le futur enseignant plus qu'à leur transmission.

La Loi d'orientation et de programme pour l'avenir de l'école de 2005 (dite loi Fillon) et surtout le Rapport qui lui est annexé ${ }^{8}$ peuvent être interprétés comme un prolongement du mouvement d'unification et d'universitarisation évoqué plus haut. En tout cas, la réforme des IUFM qu'elle induit n'a pas mis fin aux IUFM en tant que tels, malgré des mises en cause régulières et souvent passionnées, venues d'horizons politiques et de sensibilités très divers. Le trait commun de ces dénonciations, outre un fond de nostalgie et un sentiment de dégradation par rapport aux situations prévalant antérieurement, est de déplorer la baisse de qualité des savoirs et d'en attribuer la cause au "pédagogisme » et aux excès du constructivisme, saisis comme idéologie démagogique, voire criminelle aux yeux des plus virulents.

En ce qui concerne la formation des maîtres, les dispositions réglementaires adoptées dans le prolongement de la loi de 2005 ont été marquées par une modification radicale du statut des IUFM et par l'adoption du principe de " mastérisation ", impliquant que tous les enseignants recrutés par concours en France soient titulaires d'un master, soit d'un diplôme à bac +5 . On voit ici le signe concret de la poursuite de l'objectif d'unification des métiers de l'enseignement, de la tendance à l'allongement de la durée des études, de la reconnaissance de l'importance du métier, ainsi qu'une volonté de créer les conditions de la réalisation d'un espace éducatif européen. Reste à définir la nature de ces masters, et surtout, on le verra, leur articulation avec la procédure pratiquement inchangée des concours de recrutement.

$\mathrm{Au}$ plan statutaire, les IUFM ont cessé à partir de 2007 d'être des établissements publics administratifs pour devenir des «écoles internes » dotées d'une autonomie de fonctionnement assez large au sein de leurs universités de rattachement, soit une par académie. Mais, devenus une simple composante de leur université d'appartenance, les IUFM dépendent de ces dernières pour les décisions les plus importantes : dispositifs pédagogiques, budget et recrutement des personnels notamment. L'autonomie accrue et l'élargissement des compétences des universités, conséquence de la loi du 10 août 2007 relative aux libertés et responsabilités des universités, tend de fait à éloigner les IUFM de la sphère d'intervention directe de l'État. Certes ce dernier demeure dans une position tutélaire puisqu'il a établi un cahier des charges ${ }^{9}$ et qu'il est en définitive à la fois l'employeur et le maître des modalités de recrutement et de titularisation des futurs professeurs. Toutefois, le nouvel équilibre et la distribution des rôles entre l'État employeur, les universités d'appartenance mais aussi les autres

8. http://media.eduscol.education.fr/file/editorial/38/2/rapportannexe_114382.pdf.

9. Arrêté du 19 décembre 2006, JORF du 28 décembre 2006, BOEN du 4 janvier 2007. http://www.education.gouv.fr/ bo/2007/1/default.htm 
universités et les IUFM, est loin d'être établi. Les incertitudes et les contraintes auxquelles sont soumis les IUFM sont par ailleurs accrues par la complexité du passage d'une formation théorique à bac +3 à une formation professionnelle intégrée à bac +5 et par la phase d'universitarisation (au sens plein du terme) qui prévaut actuellement. Quant aux impératifs budgétaires et à la réduction du nombre de postes de fonctionnaires, particulièrement sensible dans le plus important secteur de la fonction publique d'État, ils ont pour effet de rendre les équilibres en devenir plus instables encore. De surcroît, l'articulation entre formation théorique et formation professionnelle est particulièrement complexe dans le cas du métier d'enseignant.

\section{FORMATION PROFESSIONNELLE, DIPLÔMES ET CONCOURS}

Bien que son principe ait fait l'objet d'un large consensus, ce n'est qu'à la rentrée 2010-2011 que le nouveau dispositif de formation des maîtres à bac +5 entre en application. En effet, au-delà de difficultés pratiques et administratives, les réticences et protestations convergentes manifestées au cours de l'année 2008-2009 face à la réforme des universités comme à celle de la mise en œuvre de la formation des maîtres ont conduit à un report d'une année de la réforme et à certaines modifications substantielles de l'architecture initialement projetée. Et, à l'évidence, on demeure encore assez largement dans une phase d'adaptation et de tâtonnements expérimentaux.

C'est que les problèmes à résoudre sont particulièrement ardus et intriqués, même s'ils se posent avec plus ou moins d'acuité selon les universités, les différentes disciplines ou champs disciplinaires dont la relation (voire la dépendance) aux concours de recrutement est plus ou moins étroite. Les tensions les plus vives concernent les filières du second degré et, à l'intérieur de celles-ci, les disciplines littéraires, et partant les universités à dominante de sciences humaines et sociales, pour lesquelles la préparation aux concours joue un rôle très ancien et pour ainsi dire structurant ${ }^{10}$. En revanche, et au moins dans un premier temps, le développement des masters destinés aux métiers de l'enseignement élémentaire relève d'un enjeu moins aigu. En effet, il s'agit, pour les universités et leurs unités de formation et de recherche (UFR), d'une clientèle récente dont la polyvalence disciplinaire implique des compétences et des savoirfaire dont les IUFM ont jusqu'ici la quasi-exclusivité. On peut donc imaginer, mais le phénomène peut bien n’être que de courte durée, que les universités

10. Le développement de véritables facultés de lettres et de sciences et d'un vivier d'étudiants dans ces disciplines sous la III République, tout comme l'institution des maîtres de conférences et des bourses de licence (1877) et d'agrégation (1880) a été explicitement lié à la préparation des concours de recrutement des professeurs du second degré. Voir André Chervel, Histoire de l'agrégation, Contribution à l'histoire de la culture scolaire, Paris : Kimé, 1993 et Antoine Prost, « Les universités françaises de 1808 à 1968 », Académie des sciences morales et politiques, séance du lundi 26 octobre 2009, http://www.asmp.fr/travaux/communications/2009/prost.htm/. 
ayant intégré un IUFM en leur sein possèdent une avance indiscutable en termes de compétences, de personnels et de savoir-faire pour les masters destinés au premier degré. Il en va de même pour la création des masters destinés aux professeurs des filières professionnelles courtes. Et plus généralement, l'expérience des IUFM et de leurs personnels en matière de stages et de relations avec les autorités locales (rectorats et inspections académiques) en font des interlocuteurs incontournables dans ce domaine.

Au-delà de la définition puis de l'habilitation par le ministère de l'Enseignement supérieur des masters destinés aux futurs enseignants, une des principales difficultés rencontrées renvoie à la coexistence de deux logiques distinctes : celle de la délivrance d'un grade par l'université, celle du recrutement par l'employeur. Dans la plupart des formations universitaires à finalité professionnelle, en France et dans le monde, les deux opérations sont parfaitement distinctes. Le diplôme est délivré à l'étudiant par son université ou son école et celui-ci se tourne vers son futur employeur en proposant sa candidature à un emploi. Ceci vaut notamment pour les diplômes d'ingénieurs et les masters professionnels, de plus en plus nombreux et recherchés, mais pas exclusivement. Dans cette perspective, les stages sont à la fois partie intégrante et évaluée de la formation, première épreuve de la réalité du métier (le stage peut avoir un effet attractif tout autant que dissuasif), preuve des capacités d'adaptation de l'étudiant à l'exercice de son futur métier, de son aptitude à s'évaluer (pas de stage sans rapport rédigé par le stagiaire) et de sa capacité à évoluer dans le monde professionnel. Et ces stages font bien souvent office de porte d'entrée vers une première embauche, une fois délivré le diplôme. En dernière analyse, c'est évidemment à l'employeur qu'il revient de choisir ou de rebuter tel ou tel candidat à l'embauche, en fonction de l'adéquation du candidat à son projet et à ses attentes. Le moment de la sensibilisation aux différents aspects du métier (le stage), celui de la délivrance du diplôme et celui du recrutement sont donc, au moins sur le plan théorique, trois temps distincts.

Dans la logique de la tradition éducative française, et notamment dans le cas des études littéraires au sens large pour lesquels les métiers de l'enseignement du second degré restent le principal, le plus "naturel » et le plus valorisé des débouchés aux yeux des universitaires, le concours, preuve ultime des capacités de l'étudiant, a jusqu'ici dans les faits revêtu une triple fonction. Il est perçu en effet tout à la fois comme délivrance symbolique d'une distinction universitaire qui sanctionne une ou plusieurs années de préparation spécifique très exigeante (le CAPES et plus encore l'agrégation sont très couramment, et bien évidemment à tort, assimilés à des grades universitaires), comme certification des capacités à enseigner (connaître, c'est transmettre) et comme recrutement proprement dit. Malgré les progrès indiscutables que les IUFM ont entraînés en matière de professionnalisation de la formation aux métiers des enseignants du second degré, la partie théorique du concours, restée très 
largement fondée sur les approches et les savoirs académiques, apparaît aujourd'hui encore comme l'essentiel aux yeux des usagers. Et la validation professionnelle comme une formalité : moins de $1 \%$ des stagiaires se voient refuser leur titularisation à la suite de cette année de formation.

Faute de pouvoir distinguer radicalement formation, délivrance de grade et recrutement, ce qui aurait été sans doute difficilement supportable aux yeux de l'opinion comme à ceux de la plupart des acteurs de l'éducation en France, le dispositif adopté apparaît comme le résultat de plusieurs compromis entre une série d'exigences contradictoires.

Un des reproches les plus constants, et les plus fondés, que l'on a pu adresser à l'ancien mode de recrutement des professeurs s'appuyait sur le fait que se préparer et échouer honorablement à un concours, dont le nombre de places est par définition limité et variable d'une année à l'autre, n'est pas démériter. Que vaut le fait d'avoir préparé pendant deux années avec sérieux un concours sélectif, souvent même très sélectif, et d'avoir échoué deux fois, fût-ce d'un cheveu ? La règle du concours est justement celle du tout ou rien : on est reçu ou collé, élu ou réprouvé. Sur le plan intellectuel et humain, mais aussi en termes d'intérêt général, une telle règle n'est acceptable qu'à la condition que les talents et l'investissement du candidat de valeur qui a échoué au concours puissent être reconnus et valorisés par la suite ${ }^{11}$. La définition des principes d'organisation des masters suppose donc que, destinés aux métiers de l'enseignement, ils ne leur soient pas exclusivement dédiés, et que la dimension professionnalisante soit progressive à mesure de la scolarité, allant de l'exploration et de l'observation à une participation accrue jusqu'à la prise de la classe en responsabilité. Ce que stipule explicitement la circulaire sur les masters en date du 23 décembre $2009^{12}$. Un étudiant inscrit dans un tel master doit donc pouvoir, en cours de cycle, se réorienter et valider son cursus universitaire même en cas d'échec au concours - à l'oral comme à l'écrit -, à condition naturellement que son travail soit satisfaisant. Ce postulat, parfaitement sain (que faire d'une cohorte d'étudiants reçus/collés ?), a eu plusieurs conséquences, créant autant de défis auxquelles les universités et le système éducatif se doivent de répondre.

\section{UN DÉFI POUR LES UNIVERSITÉS ET LE SYSTÈME ÉDUCATIF}

Un des premiers effets de cette organisation touche à la vie des universités elles-mêmes et, avec des variations selon les champs disciplinaires, à l'organisation des masters dans leur ensemble. En ce qui concerne la deuxième année des masters, il devient dans bien des cas de plus en plus difficile de

\footnotetext{
11. Ce qui n'était jusqu'ici que très marginalement le cas, pour les seuls candidats déjà certifiés et, selon l'expression consacrée, « bi-admissibles » à l'agrégation.

12. Circulaire $n^{\circ}$ 2009-1037 du 23-12-2009, http://www.enseignementsup-recherche.gouv.fr/pid20536/rubriquebo.html?cid_bo=50134/.
} 
distinguer de manière absolue master « recherche » et master " professionnel ». Dans les disciplines littéraires notamment, ce n'est qu'après l'agrégation que les candidats au doctorat cherchaient éventuellement à s'inscrire en thèse. Désormais, il faudra inventer des parcours spécifiques et raisonnables en temps exigé, d'autant que, paradoxalement, bien des étudiants non admissibles au concours seront tentés de terminer leur master en s'orientant en cours d'année vers une dimension recherche. Il est vrai que cette tendance à valoriser le " professionnel » face à la « recherche » était déjà à l'œuvre dans de nombreux cas, d'autant que les débouchés du doctorat se révèlent plus aléatoires que ceux des masters, comme le rappelle une récente note du Centre d'analyse stratégique ${ }^{13}$.

Une autre conséquence, sauf dans le cas où le candidat au concours est déjà titulaire d'un master, est de conduire à enchevêtrer sans les confondre le calendrier du concours de recrutement et celui du master. En effet, en règle générale, l'étudiant candidat sera à la fois inscrit au concours et en deuxième année de master, et ne pourra être recruté qu'une fois le master délivré. Dans cette perspective, pour éviter de reconduire le schéma antérieur (une année de préparation exclusivement académique suivie d'une année de formation professionnelle), la date des épreuves d'admissibilité a été fixée en automne (respectivement septembre et novembre pour les professeurs des écoles et personnels du second degré) et celle de l'admission en mai ou juin ${ }^{14}$. Ce qui implique que le rythme du master et celui du concours ne sont que partiellement disjoints et que seuls les admissibles pourront suivre les stages en responsabilité du deuxième semestre de la seconde année de master. Par ailleurs, de même que les masters font alterner formation académique et initiation au métier, les concours ont vu leur organisation modifiée. Au-delà d'une réduction et d'une simplification du nombre des épreuves ${ }^{15}$, a été introduite, pour l'ensemble des concours de recrutement des professeurs, une question d'oral impliquant chez les candidats une réflexion sur la compétence "Agir en fonctionnaire de l'État et de façon éthique et responsable », qui était inscrite dans le cahier des charges de 2006 évoqué plus haut.

Cette dernière disposition fait l'objet de contestations et de mises en cause de la part de nombreux universitaires participant à la préparation et aux jurys des concours. Au-delà des appréciations individuelles, dont beaucoup soulignent le risque d'une dérive vers une police des esprits, on peut estimer que

13. "Si l'insertion des diplômés en France augmente avec le niveau de formation, il existe depuis le début des années 2000 une césure au niveau du diplôme le plus élevé de la formation universitaire : alors qu'en 2007 les jeunes diplômés de niveau master ont un taux de chômage de 7 \%, celui des titulaires d'un doctorat culmine à 10 \%. " Centre d'analyse stratégique, Note de veille 189, juillet 2010. http://www.strategie.gouv.fr/IMG/pdf/Notedeveille189_ Emploi_des_docteurs.pdf

14. La tradition (et la réglementation) française des concours de recrutement s'appuie sur une sélection en deux temps : celle l'écrit dite de l'admissibilité, celle de l'oral ou de l'admission. L'ensemble des notes de l'écrit et de l'oral ont généralement le même poids en termes de coefficient et le ratio entre les admissibles et les admis oscille généralement entre 2 et 2,5 .

15. Arrêtés du 28 décembre 2009, JORF nº 0004 du 6 janvier 2010. 
ce qui a également choqué renvoie à une interrogation plus générale et pour ainsi dire plus professionnelle. Il en va sans doute de la conception collective que les universitaires se font de leur rôle et de leur rapport au savoir. En effet, cette épreuve induit nécessairement une dimension pédagogique ou concrète en relation directe avec l'expérience du candidat lors de ses stages, évidemment éloignée des savoirs théoriques et académiques. On voit que ce qui est en jeu ici renvoie à une question beaucoup large: quels sont les savoirs, connaissances, compétences, aptitudes et comportements à retenir lors du choix d'un candidat destiné à être professeur ? Le concours est toujours vu comme le moyen de trier et classer des têtes bien faites et bien pleines, susceptibles d'esprit d'examen et de critique, mais pas nécessairement des professeurs confrontés à la réalité souvent ardue et tendue d'un métier dont chacun s'accorde à penser qu'il est plus difficile à exercer aujourd'hui qu'hier.

Qu'est-il nécessaire de savoir pour enseigner et comment transmettre ces connaissances? On réduit trop souvent cette question à une simple opposition entre les connaissances académiques (la science, le savoir) et la pédagogie, réduite à une idéologie ou à une série de recettes pratiques et comportementales de bon sens face à une classe ou un groupe d'élèves. Or ce qui manque à notre formation des maîtres, ce n'est ni la solidité théorique ni les capacités pratiques et le dévouement des professeurs dans l'exercice de leur métier. C'est plutôt une réflexion sur le passage entre les connaissances académiques et ce qu'il est possible et bon d'enseigner. Ce qui manque le plus, c'est la dimension didactique qui ne peut exister en dehors des disciplines elles-mêmes et la capacité à imaginer non pas la seule simplification des connaissances destinées aux élèves mais leur transmission ou, pour reprendre une expression consacrée, leur «transposition ». Ce qui implique une maîtrise théorique et une réflexion approfondies sur ce qui est "transmissible» dans une situation donnée face à un public donné qu'il s'agit d' "élever », dans tous les sens du terme. Jusqu'ici, dans son ensemble, l'Université française s'est peu penchée sur cette dimension pourtant essentielle de la formation des maîtres et de la transmission des savoirs. En établissant une nouvelle relation entre les universités et la formation des maîtres, la réforme en cours peut, souhaitons-le, contribuer à modifier cette tendance en introduisant la dimension didactique dans les masters débouchant sur les métiers de l'enseignement.

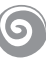

Le passage à une formation universitaire professionnalisante de cinq ans pour tous les enseignants impliquait, tant pour des raisons financières que pour éviter une entrée trop tardive dans la carrière, de ne pas reconduire à l'identique l'année de formation après le concours en vigueur dans l'ancien dispositif. Dans ces conditions, malgré des dispositifs d'allégement prévus et 
modulables localement, la première année d'exercice des néo-titulaires risque d'être particulièrement exigeante. Et, de manière plus radicale, les stages des nouveaux reçus aux concours ayant traditionnellement contribué à dégager des moyens de remplacement propres à assurer une part importante de la formation continue de leurs collègues en exercice, cette dernière risque d'en être affectée.

La formation continue des enseignants, par ailleurs, constitue une des principales faiblesses du système éducatif français, et explique pour une part, au-delà de cas individuels remarquables, les médiocres perspectives d'une véritable carrière pour les professeurs ${ }^{16}$. Les causes d'un tel mal sont évidemment nombreuses et anciennes. Au-delà des limites matérielles et budgétaires, il en est une, sans doute très française, et passablement idéologique. Il s'agit du mythe d'une formation initiale dans laquelle toute la théorie nécessaire à une activité professionnelle s'apprendrait avant qu'on ne soit conduit à l'exercer. Ce mythe a un corollaire : l'exercice et l'expérience réelle de la variété du métier ne pourraient en définitive que révéler des pratiques et des manières de faire. La réforme en cours de la formation initiale a le mérite de bousculer le premier mythe. On peut espérer que le second puisse être bousculé à son tour, permettant ainsi aux enseignants d'assurer leur progression théorique et didactique au long de leur carrière.

16. Ce que relève notamment le Livre vert remis au ministre de l’Éducation en 2008 par Marcel Pochard, conseiller d'État et ancien directeur général de l'administration et de la fonction publique. http://media.education.gouv.fr/file/ Commission_Pochard/18/8/Rapport_+_couverture_-_12-02-08_23188.pdf 
